\title{
Automated Discovery of Relationships, Models, and Principles in Ecology
}

\section{Cardoso, Pedro}

2020-12-11

Cardoso , P , Branco , V V , Borges , P A , Carvalho , J C , Rigal , F, Gabriel , R, Mammola , S , Cascalho , J \& Correia , L 2020 , ' Automated Discovery of Relationships, Models, and Principles in Ecology ' , Frontiers in Ecology and Evolution , vol. 8 , 530135 . https://doi.org/10.3389/fevo.2020.53013

http://hdl.handle.net/10138/324096

https://doi.org/10.3389/fevo.2020.530135

cc_by

publishedVersion

Downloaded from Helda, University of Helsinki institutional repository.

This is an electronic reprint of the original article.

This reprint may differ from the original in pagination and typographic detail.

Please cite the original version. 


\section{OPEN ACCESS}

Edited by:

Miguel A. Fortuna,

Estación Biológica de Doñana

(EBD), Spain

Reviewed by:

Carlos J. Melián

Swiss Federal Institute of Aquatic Science and Technology, Switzerland

Shai Pilosof,

Ben-Gurion University of the

Negev, Israel

*Correspondence:

Pedro Cardoso

pedro.cardoso@helsinki.fi

Specialty section:

This article was submitted to Models in Ecology and Evolution,

a section of the journal

Frontiers in Ecology and Evolution

Received: 28 January 2020 Accepted: 23 November 2020 Published: 11 December 2020

Citation:

Cardoso P, Branco W, Borges PAV, Carvalho JC, Rigal F, Gabriel R,

Mammola S, Cascalho J and Correia L (2020) Automated Discovery of Relationships, Models, and Principles in Ecology.

Front. Ecol. Evol. 8:530135. doi: 10.3389/fevo.2020.530135

\section{Automated Discovery of Relationships, Models, and Principles in Ecology}

\author{
Pedro Cardoso ${ }^{1,2 *}$, Vasco V. Branco ${ }^{1,3}$, Paulo A. V. Borges ${ }^{2}$, José C. Carvalho ${ }^{1,2,4}$, \\ François Rigal ${ }^{2,5}$, Rosalina Gabriel ${ }^{2}$, Stefano Mammola ${ }^{1,6}$, José Cascalho ${ }^{7}$ and \\ Luís Correia ${ }^{3}$

\begin{abstract}
1 Laboratory for Integrative Biodiversity Research (LIBRe), Finnish Museum of Natural History Luomus, University of Helsinki, Helsinki, Finland, ${ }^{2}$ CE3c - Centre for Ecology, Evolution and Environmental Changes/Azorean Biodiversity Group, Departamento de Ciências Agrárias, Universidade dos Açores, Angra do Heroísmo, Portugal, ${ }^{3}$ Laboratório de Sistemas Informáticos de Grande Escala, Faculdade de Ciências, Universidade de Lisboa, Lisbon, Portugal, ${ }^{4}$ Molecular and Environmental Centre - Centre of Molecular and Environmental Biology, Department of Biology, University of Minho, Braga, Portugal, ${ }^{5}$ Institut Des Sciences Analytiques et de Physico Chimie pour L'environnement et les Materiaux UMR5254, Comité National de la Recherche Scientifique - University de Pau et des Pays de l'Adour - E2S UPPA, Pau, France, ${ }^{6}$ Molecular Ecology Group (MEG), Water Research Institute, National Research Council, Verbania Pallanza, Italy, ${ }^{7}$ Departamento de Ciências Agrárias, Núcleo de Investigação e Desenvolvimento em e-Saúde (NIDes), Angra do Heroísmo, Portugal
\end{abstract}

Ecological systems are the quintessential complex systems, involving numerous high-order interactions and non-linear relationships. The most used statistical modeling techniques can hardly accommodate the complexity of ecological patterns and processes. Finding hidden relationships in complex data is now possible using massive computational power, particularly by means of artificial intelligence and machine learning methods. Here we explored the potential of symbolic regression (SR), commonly used in other areas, in the field of ecology. Symbolic regression searches for both the formal structure of equations and the fitting parameters simultaneously, hence providing the required flexibility to characterize complex ecological systems. Although the method here presented is automated, it is part of a collaborative human-machine effort and we demonstrate ways to do it. First, we test the robustness of SR to extreme levels of noise when searching for the species-area relationship. Second, we demonstrate how SR can model species richness and spatial distributions. Third, we illustrate how SR can be used to find general models in ecology, namely new formulas for species richness estimators and the general dynamic model of oceanic island biogeography. We propose that evolving free-form equations purely from data, often without prior human inference or hypotheses, may represent a very powerful tool for ecologists and biogeographers to become aware of hidden relationships and suggest general theoretical models and principles.

Keywords: artificial intelligence, ecological complexity, evolutionary computation, genetic programming, species richness estimation, species-area relationship, species distribution modeling, symbolic regression

\section{INTRODUCTION}

Complexity is a term often used to characterize systems with numerous components interacting in ways such that their collective behavior is difficult to predict, but where emergent properties give rise to patterns, more or less simple but seldom linear (Table 1) (Holland, 1995; Mitchell, 2009). Complex systems science is therefore an effort to understand non-linear systems with multiple 
TABLE 1 | Glossary of terms.

Artificial Intelligence (AI)-A scientific field concerned with the automation of activities we associate with human thinking (Russell et al., 2010).

Big data-Very large amount of structured or unstructured data, hard to model with general statistical techniques but with the potential to be mined for information.

Complex system-A system in which a large network of components organize, without any central controller and simple although non-linear rules of operation, into a complex collective behavior that creates patterns, uses information, and, in some cases, evolves, and learns (Mitchell, 2009).

General model-An equation that is found to be useful for multiple datasets, often but not necessarily, derived from a general principle. In most cases the formal structure of equations is kept fixed, while some parameters must be fitted for each individual dataset.

General principle-Refers to concepts or phenomenological descriptions of processes and interactions (Evans et al., 2013). May not have direct translation to any general model, but be a purely conceptual abstraction.

Genetic programming (GP)-A biologically-inspired method for getting computers to automatically create a computer program to solve a given problem (Koza, 1992). It is a type of evolutionary algorithm, where each solution to be tested (individual in a population of possible solutions) is a computer program.

Pareto front-A curve connecting a set of best solutions in a multi-objective optimization problem. If several conflicting objectives are sought (e.g., minimize both error and complexity of formulas), the Pareto front allows visualizing the set of best solutions (Smits and Kotanchek, 2005).

Symbolic regression (SR)-A function discovery approach for modeling of multivariate data. It is a special case of genetic programming, one where possible solutions are equations instead of computer programs.

connected components and how "the whole is more than the sum of the parts" (Holland, 1998). Biological systems probably are among the most complex (Solé and Goodwin, 2000), and among them, ecological systems are the quintessential complex systems (Anand et al., 2010). These are composed of individuals from different species, interacting and exchanging energy in multiple ways, furthermore, relating with the physical environment at different spatial and temporal scales in nonlinear relationships. Consequently, ecology is dominated by idiosyncratic results, with most ecological processes being contingent on the spatial and temporal scales in which they operate. This makes it difficult to identify recurrent patterns, knowing also that pattern does not necessarily identify process (Lawton, 1996; Dodds, 2009; Passy, 2012). The most used exploratory (e.g., principal component analysis) and statistical modeling techniques (e.g., linear and non-linear regressions) can hardly reflect the complexity of ecological patterns and processes, often failing to find meaningful relationships in data. More flexible techniques, such as generalized additive models (GAMs), usually do not allow an easy interpretation of results and particularly of putative causal relationships (e.g., Sugihara et al., 2012). For ecological data, we require more flexible and robust, yet amenable to full interpretation, analytical methods, which can eventually lead to the discovery of general principles and models.

The aim of any ecological principle is to provide a robust model for exploring, describing, and predicting ecological processes regardless of taxon identity and geographic region (Lawton, 1996; Dodds, 2009). Finding a recurrently high goodness-of-fit for a model to an ecological pattern for most taxa and ecosystems is usually a compelling evidence of a mechanistic process controlling that pattern. When general principles are translated into robust models, general statistical methods are mostly abandoned in favor of these, of which only few examples exist in ecology (Data Sheet $\mathbf{1}$ ). Such general, widely applicable equations are mostly found by intellectual tour de force. Yet, they are only the tip of the iceberg, usually incorporating few of the variables increasingly available to ecologists and that could potentially explain such patterns.

The automation of techniques for collecting and storing ecological and related data, with increasing spatial and temporal resolutions, has become one of the central themes in ecology and bioinformatics. Yet, automated and flexible ways to synthesize such complex and big data were mostly lacking until recently (Martin et al., 2018; Chen et al., 2019; Desjardins-Proulx et al., 2019). Finding hidden relations within such data is now possible using massive computational power. New computer-intensive methods have been developed or are now available or possible (Reshef et al., 2011), including the broad field of artificial intelligence (AI) or machine learning (ML) which have produced a variety of approaches (Lu, 2019). Artificial intelligence includes a series of evolution-inspired techniques, brought together in the sub-field of evolutionary computation, of which the most studied and well-known probably are genetic algorithms (Holland, 1975). Genetic programming, namely in the form of symbolic regression (SR) (Koza, 1992), is a derivation of genetic algorithms that searches the space of mathematical equations without any constraints on their form. Hence, it provides the required flexibility to represent complex systems as presented by many ecological systems (Figure 1). Contrarily to traditional statistical techniques, symbolic regression searches for both the formal structure of equations and the fitting parameters simultaneously (Schmidt and Lipson, 2009). Finding the structure of equations is especially useful to discover general models, providing insights into the processes and eventually leading to the discovery of new and yet undiscovered principles. Fitting the parameters provides insight into the raw data and allow for specific predictions. Successful examples on the use of SR in ecology include modeling of land-use change (Manson, 2005; Manson and Evans, 2007), effects of climate change on populations (Tung et al., 2009; Larsen et al., 2014), community distribution (Larsen et al., 2012; Yao et al., 2014), predicting micro-organismal blooms (Muttil and Lee, 2005; Muttil and Chau, 2006; Jagupilla et al., 2015; Tromas et al., 2017), deriving vegetation indices (Almeida et al., 2015), forecasting the trophic evolution of lakes (Bertoni et al., 2016), using parasites as biological tags (Barrett et al., 2005), and even to revisit classical ecological models such as the Lotka-Volterra predator-prey equation (Martin et al., 2018; Chen et al., 2019).

The goal of this work is to explain, test, and show the usefulness of SR in uncovering hidden relationships within typical ecological datasets. To illustrate this, we used five case studies reflecting typical analytical problems faced by ecologists. In the first example, (i) we test the robustness of SR when finding the power law applied to the species-area relationship (SAR) with 


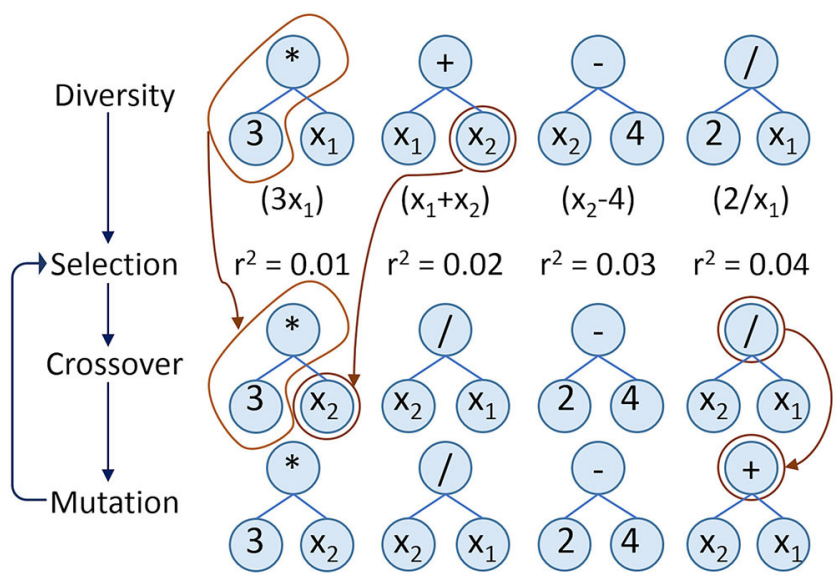

FIGURE 1 | Schematic representation of the symbolic regression workflow. The basic representation is a parse-tree where building blocks such as variables (in this case: $x_{1}, x_{2}$ ), parameters (integers or real numbers), and operators (e.g.,,,$+- \times, \div$ ) are connected forming functions (in parenthesis under the first line of trees). Initial equations are generated by randomly linking different building blocks. Equations are combined through crossover, giving rise to new equations with characteristics from both parents (arrows linking the first and second rows of trees). Equations with better fitness (e.g., $R^{2}$ ) have higher probabilities of recombining. To avoid loss of variability, a mutation step is added after crossover (arrows linking the second and third rows of trees). After multiple generations, evolution stops and a set of free-form equations best reflecting the input data is found.

extreme levels of noise, even beyond the high levels typical of ecological datasets. In the next two examples, we demonstrate how SR can deal with complex datasets, namely to model (ii) species richness; and (iii) species spatial distributions. Finally, we illustrate how SR can be used to find general models in ecology, by using it to develop new formulas for (iv) species richness estimation; and v) the general dynamic model of oceanic island biogeography (GDM).

\section{GENERAL METHODOLOGY}

Symbolic regression works as a computational parallel to the evolution of species. A population of initial equations is generated randomly by combining different building blocks, such as the variables of interest (independent explanatory variables), algebraic operators (e.g.,,,$+- \div, \times$ ), analytic function types (exponential, log, power, etc.), constants and other ways to combine the data (e.g., Boolean or decision operators) (Figure 1). Being random, these initial equations almost invariably fail in describing the patterns or phenomena of interest, but some equations are slightly better than others. All are then combined through crossover, giving rise to new equations with characteristics from both parents. Equations with better fitnessas estimated using a chosen statistical measure such as $R^{2}$ or Akaike's Information Criterion (AIC; Akaike, 1974)-have a higher probability of recombining. To avoid new equations being bounded by initially selected building blocks or quickly losing variability along the evolutionary process, a mutation step (acting

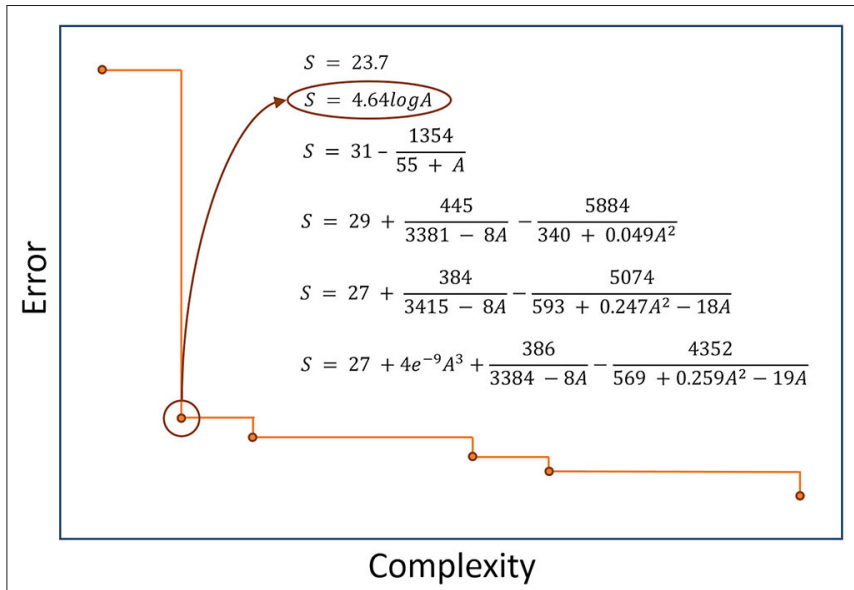

FIGURE 2 | Example of a Pareto front depicting error vs. complexity. This example reflects a symbolic regression search of the best species-area relationship for native spiders in the Azores (Portugal). The second formula is clearly the most promising, with both high accuracy (low error) and low complexity. In many occasions a single formula is not clearly best, in which case weights can be given to each of them through indices that simultaneously positively weight accuracy and negatively weight complexity (such as AIC or $\mathrm{BIC}$ ) and/or multiple formulas presented as possible outcomes.

on any building block) is added to the process after crossover. After multiple generations, an acceptable level of accuracy by some of the equations is often attained and the researcher stops the process.

For this work we used the software Eureqa (Nutonian, Inc) (Schmidt, 2015), which provides an intuitive interface suitable also for non-expert SR users (Dubčáková, 2011). Although a commercial version is available, we used the freely available test version for all analyses. For each run, the software outputs a list of equations along an error/complexity Pareto-front (Smits and Kotanchek, 2005), with the most accurate equation for each level of complexity being shown (Figure 2). For the SR search we used only algebraic and analytic operators $(+,-, \div, \times, \log$, power) in all examples below, so that outputs could be most easily interpreted. The goodness-of-fit was evaluated using $R^{2}$ or AICc, depending on the question (see below). The Pareto-front often presents an "elbow," where near-minimum error meets nearminimum complexity. The equation in this inflection is closer to the origin of both axes and is a good starting point for further investigation-if both axes are in comparable qualitative scales. Often, however, this inflection point is not obvious, and a single formula is not clearly best. In such cases, weights can be given to each of them through indices that positively weight accuracy and negatively weight complexity, such as AICc (Akaike, 1974). However, in all cases it is important to check all formulas along the Pareto-front. Often equations or models that make immediate sense to the specific question may not be detected by these automated methods.

Each of the five case studies was analyzed independently and using different approaches to test the performance of SR against other methods. We often opted to use fully independent datasets for three reasons. First, we were looking for general formulas, 
which should be tested against fully independent data. Second, it was a much more powerful and convincing way to demonstrate the method than using subsets of the same data, which necessarily have some common ground that facilitates the job of any method. Third, our datasets, as typical for many in ecology, often had very few observations, making it hard to do sub-setting in few cases.

\section{CASE-STUDIES}

\section{Finding the Species-Area Relationship (SAR) With Extreme Levels of Noise}

Typical ecological datasets not only have few data points, but these are prone to varying levels of noise. Noise can be due to natural phenomena, such as spillover from neighboring regions, unpredictable weather events, etc., lack of the most appropriate data to model the phenomena, or simply errors in measuring or sampling. Testing any novel method to its robustness to different sources and levels of noise is therefore imperative.

One of the most studied examples of SARs is their application to island biogeography (ISAR). The shape of ISARs has been modeled by many functions, but three of the simplest seem to be preferred in most cases, the power, exponential, and linear models (Triantis et al., 2012) (Data Sheet 1). The power model in particular includes island area (A) and two fitting parameters, $c$ (the intercept) and $z$ (the scaling of richness with area) (Data Sheet 1). Here we created 30 fictional islands each one corresponding to one of the 30 possible combinations resulting from five different areas $\left(10,100,1000,10,000\right.$, and 100,000 $\left.\mathrm{km}^{2}\right)$, two typical values for $c$ (1 and 10), and three typical values for $z$ $(0.2,0.3$, and 0.4$)$. We then simulated sampling from these 30 islands, each with a sampled richness equal to the multiplication of the true richness value by five different levels of noise as given by the standard deviation of a sampling from a normal distribution with mean $=1$ and $\mathrm{sd}=0,0.1,0.2,0.4$, and 0.8 . The theoretical richness of each island was then multiplied by 10 simulations of each noise level using this approach, providing a total of 50 search trials (Data Sheet 2). We must emphasize that with sd $=0.8$ the noise was extreme and unreasonable, with for example islands predicted to have 100 species presenting anything between 0 and 199 species after noise was added. We evaluated the ability of SR to develop the power law by counting at each noise level how many times the usual formulation and a derivation without the intercept $c$ were found among the 10 searches per level.

Our simulations using SR were able to find the power-law of the SAR even with the most extreme scenarios (Data Sheet 2). From $100 \%$ success rate with sd $=0$ or 0.1 , to $70 \%$ with sd $=$ $0.2,50 \%$ with sd $=0.4$, and $40 \%$ with sd $=0.8$. If we include the simpler formulation with no intercept, success rate was 100, 100, 100,90 , and $80 \%$, respectively for $\mathrm{sd}=0,0.1,0.2,0.4$, and 0.8 .

\section{Modeling Species Richness}

Modeling and mapping the species richness of high diversity taxa at regional to large scales is often impossible without extrapolation from sampled to non-sampled sites. Here, we used an endemic arthropod dataset collected in Terceira Island, Azores. Fifty-two sites were sampled using pitfall traps for epigean arthropods (Cardoso et al., 2009), 13 in each of four land-use types: natural forest, exotic forest, semi-natural pasture, and intensively managed pasture. In this problem, given the size of the dataset, we used a 5-fold cross validation. We explained and predicted species richness per site using elevation, slope, annual average temperature, annual precipitation, and an index of disturbance with values ranging from 0 (absence of human presence) to 100 (dense urban environment) (Cardoso et al., 2013). For SR we ran each fold five times to minimize the risk that the formulas found represented local optima. We then reported the average and range of $R^{2}$ and AICc of the five partitions for both the training and test data.

As the response variable was count data, Generalized Linear Models (GLM) and Generalized Additive Models (GAM) with a Poisson error structure and a log link were used. We used the package MuMIn (Barton, 2015) and the $R$ environment ( $R$ Core Team, 2015) for multi-model inference based on AICc (Hurvich and Tsai, 1989) values, using all variables plus all possible interactions for GLM. For fitting GAM, we used package gam (Hastie, 2015). The $R^{2}$ goodness of fit was used as the fitness measure. For each run of the SR (25 in total) we picked the formula at the inflection point of the Pareto-front (Data Sheet 2). Both $R^{2}$ and AICc were used to compare GLM and GAM with SR on the test datasets.

The model selected by GLM in all five $k$-folds was:

$$
S=e^{(\mathrm{a}+\mathrm{b} H-\mathrm{c} P-\mathrm{d} D)}
$$

where $H=$ altitude, $P=$ precipitation, $D=$ disturbance; $\mathrm{a}, \mathrm{b}$, $c$, and $\mathrm{d}$ are fitting parameters with mean $\mathrm{a}=1.894$ (range: $1.116-2.577$ ), mean $b=0.00419$ (range: $0.00360-0.00574$ ), mean $c=0.000972$ (range: 0.000726-0.001212), and mean $\mathrm{d}=$ 0.0251 (range: $0.0118-0.0331$ ). The mean training $R^{2}=0.529$ (range: $0.469-0.573$ ) and mean training $\mathrm{AICc}=104.151$ (range: 103.055-105.634). The mean testing $R^{2}=0.528$ (range: $0.313-$ 0.770 ) and mean testing AICc $=45.340$ (range: $41.913-48.513$ ).

The results of GAM were similar to the GLM, the algorithm selecting the most parsimonious formulation equivalent to a GLM. The SR results performed considerably better than GLM or GAM with a much simpler formula using a single variable (Disturbance) and much better training and testing results, with 23 out of 25 formulas chosen being in the form:

$$
S=(a / D)-b
$$

where $\mathrm{a}$ and $\mathrm{b}$ were fitting parameters with mean $\mathrm{a}=140.787$ (range: 134.700-145.775) and mean $b=1.325$ (range: $1.078-$ 1.483). The mean training $R^{2}=0.603$ (range: 0.576-0.644) and mean training AICc $=52.631$ (range: 47.982-56.985). The mean testing $R^{2}=0.601$ (range: $0.449-0.737$ ) and mean testing $\mathrm{AICc}=19.088$ (range: 13.042-23.432). We should emphasize the simplicity of interpretation of this formula, indicating that species richness essentially was inversely related with disturbance.

\section{Modeling Species Distributions}

Species distribution modeling (SDM) is one of the most widely used correlative statistical approaches to biodiversity assessment, 


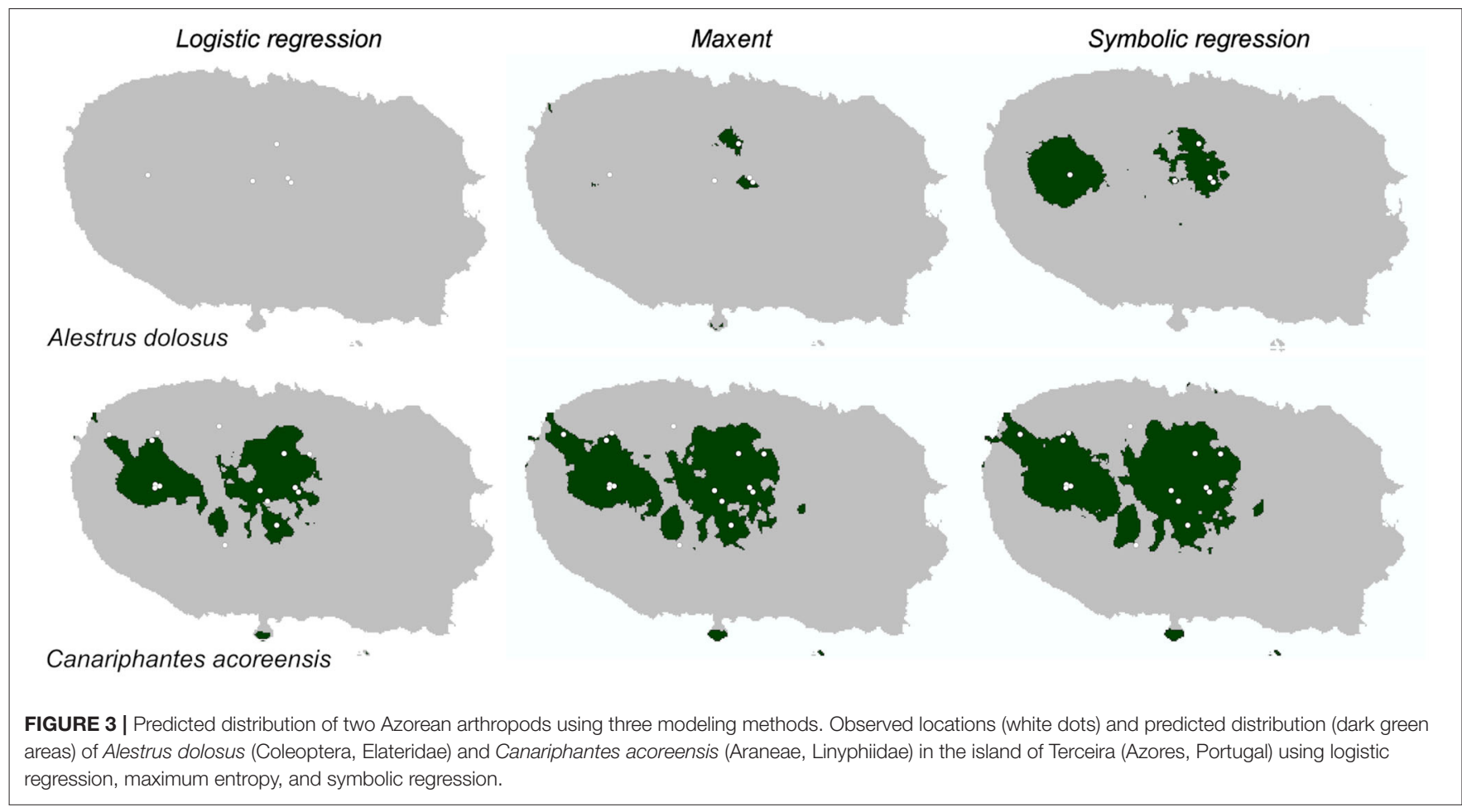

for example to fill gaps in our knowledge on individual species distributions, predict species response to climate change, and the spread of invasive species (Araújo et al., 2019). As a case study, we modeled the potential distribution of two endemic Azorean species in Terceira Island: the rare forest clickbeetle Alestrus dolosus (Crotch) (Coleoptera, Elateridae) and the abundant but mostly forest-restricted spider Canariphantes acoreensis (Wunderlich) (Araneae, Linyphiidae). We compared the performance of logistic regressions (GLM with a binomial error structure) and maximum entropy models (Maxent; Phillips et al., 2006) in predicting the distribution of the two species, to that of SR. GLM and MaxEnt are two of the most widely used approaches for SDM (Elith et al., 2006). Given the intrinsic differences between methods, we had to use different background datasets (Data Sheet 3). Maxent used the environmental maps of the islands with a resolution of $100 \mathrm{~m}$, from where it extracted pseudo-absences. We then converted the probabilistic potential distribution maps to presence/absence using the maximum value of training sensitivity plus specificity as the threshold as recommended by Liu et al. (2005). Logistic regression and SR used presence/absence data from the 52 sampled sites. We conducted multi-model inference of logistic regression based on AICc values. In the SR run we included a step function, so that positive and negative values were converted to presence and absence (binary output), respectively. Absolute error, reflecting the number of incorrect classifications, was used as the fitness measure. As inflection points of the Pareto fronts were clear, the best SR formula for each species was chosen based on them (Data Sheet 2). Given the scarcity of occurrences of species in the dataset (from 10 to $35 \%$ of the data points) we opted for a balanced split of $50 \%$ for training and test sets. In all cases only the training data (26 sites) were used to obtain the models. Logistic GLM, Maxent, and SR were compared in their performance for predicting presence and absence of species on the 26 test sites using the Sensitivity, Specificity, and True Skill Statistic (TSS) (Allouche et al., 2006).

The potential distribution models were relatively similar for $C$. acoreensis but show marked differences for A. dolosus (Figure 3). Symbolic regression outperformed both other models for A. dolosus and was as good as Maxent for C. acoreensis, with both outperforming logistic regression (Table 2). The SR models were not only the best, presenting maximum values for TSS, but were also the easiest to interpret. A. dolosus was predicted to have adequate environmental conditions in all areas above $614 \mathrm{~m}$ elevation, being restricted to pristine native forest. Canariphantes acoreensis could potentially be present in all areas with disturbance values below 41.3, occurring not only in native forest but also in adjacent semi-natural grassland and humid exotic forest. The logistic regression and Maxent models used a large number of explanatory variables for $A$. dolosus yet performed worse on the test data than did SR (Table 2).

\section{Developing Species Richness Estimators}

Several asymptotic functions have been used to estimate species richness (Soberón and Llorente, 1993), including the Clench function (Clench, 1979), the negative exponential function, and the rational function (Ratkowsky, 1990) (Data Sheet 1). We used SR to rediscover or eventually find novel asymptotic models that would outperform them. Two independent datasets were used resulting from exhaustive and standardized sampling for 
TABLE 2 | Species distribution models for two endemic arthropod species on the island of Terceira (Azores, Portugal).

\begin{tabular}{|c|c|c|c|c|}
\hline Model & Formula & Sensitivity & Specificity & TSS \\
\hline \multicolumn{5}{|l|}{ Alestrus dolosus } \\
\hline Logistic regression & $1 /\left(1+e^{-8469+0.432 P+540.7 T}\right)$ & 0 & 1 & 0 \\
\hline Maxent & Uses all variables but $S I$, main is $D$ (contribution $=74.1 \%$ ) & 0.5 & 1 & 0.5 \\
\hline Symbolic regression & step $(\mathrm{H}-614)$ & 1 & 0.75 & 0.75 \\
\hline \multicolumn{5}{|c|}{ Canariphantes acoreensis } \\
\hline Logistic regression & $1 /\left(1+e^{-3.617+0.103 D}\right)$ & 0.667 & 0.7 & 0.367 \\
\hline Maxent & Uses only $D$ (contribution $=100 \%)$ & 0.833 & 0.65 & 0.483 \\
\hline Symbolic regression & step (41.3-D) & 0.833 & 0.65 & 0.483 \\
\hline
\end{tabular}

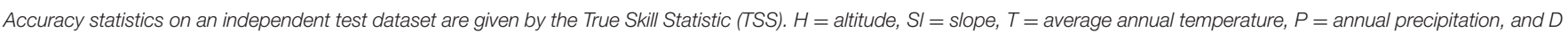

= disturbance index. The step function in symbolic regression converts positive values inside parentheses to presence and negative values to absence. Best values in bold.

spiders in 1 ha plots, performed by 8 collectors during $320 \mathrm{~h}$ of sampling in a single hectare using five different methods (so-called sampling protocol "COBRA" - Conservation Oriented Biodiversity Rapid Assessment; Cardoso, 2009). The training dataset was from a mixed forest in Gerês (northern Portugal) and the test dataset was from a Quercus forest in Arrábida (southern Portugal) (Cardoso et al., 2008a,b).

Randomized accumulation curves for both sites were produced using the R package BAT (Cardoso et al., 2015). The true diversity of each site was calculated as the average between different non-parametric estimators (Chao 1 and 2, Jackknife 1 and 2). Because the sampled diversity in the training dataset reached a very high completeness but we wanted to simulate typically very incomplete sampling, datasets with 10, 20, 40, 80, and 160 randomly chosen samples were extracted and used, in addition to the complete 320 samples dataset, as independent runs in SR. Squared error was used as the fitness measure. Additionally, we imposed a strong penalty to non-asymptotic functions, although these were still allowed in the search process (see Data Sheet 2 for details). The weighted and non-weighted scaled mean squared errors implemented in BAT (Cardoso et al., 2015) were used as accuracy measures.

For the training dataset, one asymptotic model was found by SR (Data Sheet 2):

$$
S=\frac{a Q}{b+Q}
$$

where $a$ and $b$ were fitting parameters. This model was in fact the Clench model with a different formulation (Data Sheet 1), where the asymptote was $a$. A second, slightly more complex but better fitting, model was found for partial datasets with 40 or more samples:

$$
S=\frac{c+a Q}{b+Q}
$$

where $c$ is a third fitting parameter. The asymptote was again given by the value of $a$ (Figure 4). This model was similar to the rational function (Data Sheet 1). It was found to outperform the Clench and negative exponential for both the training and testing datasets (Table 3).

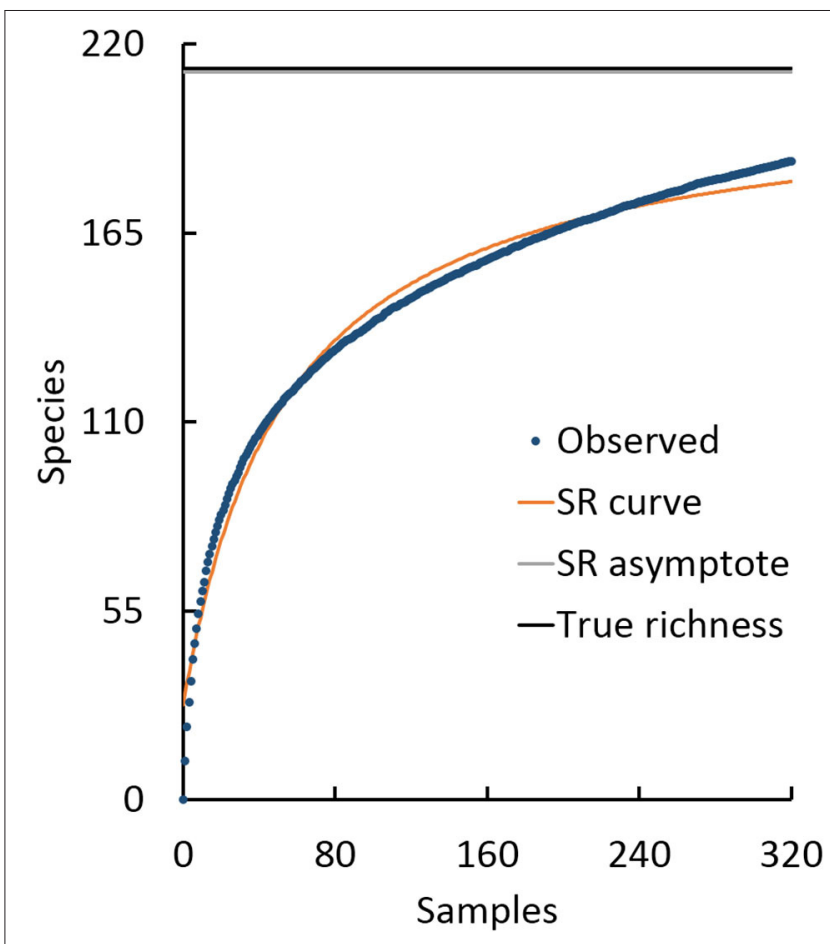

FIGURE 4 | Accumulation curve for spider sampling in Gerês (Portugal). The result of searching for the best fitting asymptotic formula using symbolic regression is also shown.

\section{Developing the General Dynamic Model of Oceanic Island Biogeography (GDM)}

The general dynamic model of oceanic island biogeography (GDM) was proposed to predict the responses of the key processes of immigration, speciation and extinction in volcanic islands, recognizing the role of geological processes in driving diversity on oceanic islands (Whittaker et al., 2008). Traditionally, the GDM is tested using a model where island species diversity is regressed as a function of area and age. Several different equations have been found to describe the GDM, extending the different SAR models with the addition 
TABLE 3 | Comparison of three asymptotic equations used to estimate spider species richness in two forest sites.

\begin{tabular}{lcc}
\hline Model & Raw accuracy & Weighted accuracy \\
\hline Gerês (training) & & \\
Observed & 0.113 & 0.037 \\
Clench & 0.055 & 0.018 \\
Negative exponential & 0.115 & 0.049 \\
Rational function & $\mathbf{0 . 0 4 5}$ & $\mathbf{0 . 0 1 2}$ \\
Arrábida (testing) & & \\
Observed & 0.103 & 0.031 \\
Clench & 0.038 & 0.010 \\
Negative exponential & 0.092 & 0.037 \\
Rational function & $\mathbf{0 . 0 3 2}$ & $\mathbf{0 . 0 0 8}$ \\
\hline
\end{tabular}

See Data Sheet 1 for formulas. Raw accuracy is the scaled mean squared error considering the entire observed accumulation curve (each formula was fitted to the curves using 4-320 samples) and weighted accuracy is this value weighted by the sampling effort at each point in the curve (where effort is the ratio between number of individuals and observed species richness). Note that lower values (in bold) are better as they reflect the deviation from a perfect estimator.

of a polynomial term using island age and its square (TT2), depicting the island's ontogeny. The first to be proposed was an extension of the exponential model (Whittaker et al., 2008), the power model extensions following shortly after (Fattorini, 2009; Steinbauer et al., 2013).

Our objective was to test if we could re-discover or eventually refine existing models for the ISAR and GDM from data alone. We used the Azores and Canary Islands spiders (Cardoso et al., 2010) as training data. To independently test the generality of models arising from spider data, we used bryophyte data from the same archipelagos (Aranda et al., 2014). The area and maximum time since emergence of each island were used as explanatory variables and the native species richness per island as the response variables (Data Sheet 4 ). The $R^{2}$ value was used as the fitness measure. The best SAR and GDM equations found by SR were chosen based on the inspection of the Pareto front (Data Sheet 2) but looking also for interpretability of the models. These were then compared with the existing models using AICc and the $R$ package BAT (Cardoso et al., 2015).

For the Azorean spiders, the best fitting previous model (both highest $R^{2}$ and lowest AICc) for the ISAR was the exponential model (Table 4). The SR run discovered roughly the same model, indicating, however, that the intercept ( $c$ term) was adding unnecessary complexity. A similar ranking of models was verified for bryophytes in the same region, revealing the robustness of the new model.

For the Canary Islands, the best model for spiders was a linear function of area:

$$
S=75+0.047 A
$$

$\left(R^{2}=0.364, \mathrm{AICc}=65.631\right)$. Although it was easy to interpret, the explained variance was relatively low. The SR run reached a much higher explanatory power:

$$
S=112-1.002^{A}
$$

$\left(R^{2}=0.806, \mathrm{AICc}=57.320\right)$. In this case though, the equation was over-fitting to the few available data (7 data points), as this function was erratic creating a biologically indefensible model. The reason the ISAR was hard to model for the Canary Islands spiders was because we were missing the major component Time (Cardoso et al., 2010). This was depicted by the GDM, of which the best of the current equations was found to be the power model described by Fattorini (2009) (Table 4). Nevertheless, using SR we were able to find an improved, yet undescribed, model (Table 4). This represented a general model expanding the linear SAR:

$$
S=c+z A+X T-y T^{2}
$$

When tested with Canarian bryophytes, this new formulation wss almost as good as the power model (Table 4).

\section{DISCUSSION}

Symbolic regression has the advantage over most standard regression methods (e.g., GLM) of being more flexible, allowing a good fitting to data with better interpretability, since results are in the form of mathematical formulas. GLMs and other similar techniques assume linear relations between variables or require a priori knowledge on the form of the relation (e.g., quadratic, cubic, interactions between variables, etc.).

SR also has one or more advantages over other, commonly used, highly flexible regression (e.g., GAMs) or machine learning techniques (e.g., neural-networks): (1) numerical, ordinal, and categorical variables are easily combined; (2) redundant variables are usually eliminated in the search process and only the most important are retained if anti-bloat measures (intended to reduce the complexity of equations) are used. Incidentally, this also releases the user from the problem of dealing with collinearity (Dormann et al., 2013); (3) the evolved equations are humanreadable and interpretable; and (4) solutions are easily applied to new data.

Using SR, we were able to "distill" free-form equations and models that not only consistently outperform but are more intelligible than the ones resulting from rigid methods, such as GLM, or "black-boxes", such as Maxent. This was the case for both species richness and distribution models.

We were also able to re-discover and refine equations for estimating species richness based on sampling curves and the ISAR and GDM from data alone. All the examples presented in this work suggest that evolving free-form equations purely from data, often without prior human inference or hypotheses, may represent an under-explored but very powerful tool for ecologists and biogeographers, allowing the finding of hidden relationships in data and suggesting new ideas to formulate general theoretical principles.

The idea that SR is a powerful tool for reverse-engineering ecological theory from data is not new. Many examples reviewed in the introduction suggest that different authors across disparate disciplines understand this date back to the early 2000s. Recently, the potential of SR in ecology was discussed in two essays that showed how SR can be used to develop classic demographic time 
TABLE 4 | Species area relationship (SAR) models for Azorean taxa and General Dynamic Models (GDM) of oceanic island biogeography for Canarian taxa.

\begin{tabular}{|c|c|c|c|}
\hline Model & Formula & $R^{2}$ & $\mathrm{AICc}$ \\
\hline \multicolumn{4}{|c|}{ SAR Azorean Spiders (training) } \\
\hline Power & $S=13.379 A^{0.438}$ & 0.642 & 32.505 \\
\hline Exponential & $S=0.549+4.538 \log A$ & 0.780 & 28.102 \\
\hline Linear & $S=19.357+0.017 A$ & 0.435 & 36.604 \\
\hline SR & $S=4.641 \log A$ & 0.780 & 23.319 \\
\hline \multicolumn{4}{|c|}{ SAR Azorean Bryophytes (testing) } \\
\hline Power & $S=181.625 A^{0.803}$ & 0.666 & 78.085 \\
\hline Exponential & $S=-27.824+57.114 \log A$ & 0.728 & 76.208 \\
\hline Linear & $S=196.215+0.259 A$ & 0.617 & 79.295 \\
\hline SR & $S=51.889 \log A$ & 0.722 & 71.617 \\
\hline \multicolumn{4}{|c|}{ GDM Canarian Spiders (training) } \\
\hline Whittaker & $S=-185.589+41.732 \log A+17.776 T-1.022 T^{2}$ & 0.873 & 110.350 \\
\hline Fattorini & $\log S=2.585+0.281 \log A+0.157 T-0.009 T^{2}$ & 0.941 & 105.025 \\
\hline Steinbauer & $\log S=3.367+0.098 \log A+1.502 \log T-0.454 \log T^{2}$ & 0.814 & 113.007 \\
\hline SR & $S=42.283+0.051 A+17.379 T-T^{2}$ & 0.952 & 61.505 \\
\hline \multicolumn{4}{|c|}{ GDM Canarian Bryophytes (testing) } \\
\hline Whittaker & $S=-176.599+66.602 \log A+21.361 T-1.620 T^{2}$ & 0.773 & 125.214 \\
\hline Fattorini & $\log S=4.544+0.137 \log A+0.126 T-0.009 T^{2}$ & 0.803 & 124.217 \\
\hline Steinbauer & $\log S=5.136+0.017 \log A+1.063 \log T-0.382 \log T^{2}$ & 0.612 & 128.963 \\
\hline SR & $S=192.660+0.075 A+20.702 T-1.576 T^{2}$ & 0.785 & 124.841 \\
\hline
\end{tabular}

$S=$ native species richness; $A=$ area of the island; $T=$ maximum time of emergence. Best models are indicated in bold.

series from data (Martin et al., 2018; Chen et al., 2019). Yet, our study is the first to bring together all these disparate applications, demonstrating the versatility of this tool by applying it to a range of diverse ecological and evolutionary problems, both theoretical and practical. These results suggest that the true potential for the use of SR in ecology and evolution is yet to be fulfilled.

\section{From Particular to General Principles}

Scientific fields such as physics rarely rely on general statistical inference methods such as linear regression for hypothesis testing. The complexity of ecology made such methods an imperative in most cases. Symbolic regression not only allows the discovery of relationships specific to particular datasets, but also the finding of general models, globally applicable to multiple systems of particular nature, as we tried to exemplify. SR has the significant advantage of generating a variety of expressions from the given function set. For example, other methods may be configured to fit a polynomial to the data, but the user has to specify the degree. In SR, the power function in addition to the four basic arithmetic operations, allows the generation of unlimited degree polynomials, therefore providing a wider exploration of the solution space.

As mentioned, SR is designed to optimize both the form of the equations and the fitting parameters simultaneously. The fitting parameters usually are specific to each dataset, but the form may give clues toward general principles. For example, all archipelagos will follow an ISAR, even if each archipelago will have its own $c$ and $z$ values. Although this aspect has not been explored in this study, we suggest two ways of finding general principles. First, as was hinted by our estimators' example, one may independently analyse multiple datasets from the same type of systems. From each dataset, one or multiple equations may arise. Many of these will be similar in form even if the fitting parameters are different. Terms repeated in several equations along the Pareto front or with different datasets tend to be meaningful (Schmidt and Lipson, 2009). We may then try to fit the most promising forms to all datasets optimizing the fitting parameters to each dataset and look for which forms seem to have general value over all data. Second, one may simultaneously analyse multiple datasets from the same type of systems but with a change to the general SR implementation. Instead of optimizing both form and fitting parameters, the algorithm may focus on finding the best form, with fitting parameters being optimized during the evaluation step of the evolution for each dataset independently. This parameter optimization could be done with standard methods such as quasi-newton or simplex (Nocedal and Wright, 1999). To our knowledge, this approach has yet to be implemented, but it would allow finding general models and possibly principles, independently of the idiosyncrasies of each dataset.

\section{The Need for Human Inference}

Many data mining techniques are regarded, and rightly so, as "black boxes." SR is transparent in this regard, as variables are related through human-interpretable formulas. This is particularly important if the goal is to find equations with both predictive and explanatory power, building the bridge between finding the pattern and explaining the driving process, or if a general principle is to be suggested. 
Our results show that an automated discovery system can identify meaningful relationships in ecological data. Yet, as shown by our Canary Island spider SAR model, some equations might be very accurate but overfit the data. As with any relationship finding, either automated or human, correlation does not imply causation, and spurious relationships are not only possible but probable given complex enough data.

Although the method here presented is automated, it is part of a collaborative human-machine effort. The possibility of exploiting artificial intelligence working together with human expertise can be traced back to Engelbart (1962), where the term "augmented intelligence" was coined to designate such collaboration. It has been subsequently developed and extended to teamwork involving one or more artificial intelligence agents together with one or (many) more humans, in diverse domains such as robotic teams (Yanco et al., 2004) or collective intelligence for evolutionary multi-objective optimization (Cinalli et al., 2015). In ecological problems, human knowledge may play a fundamental role: (1) in the beginning of the process, when selecting input variables, building blocks and SR parameters; and (2) in the interpretation and validation of equations. The choice of equations along a machine-generated Pareto front should also take advantage of human expert knowledge to identify the most interesting models to explain the data. The researcher might then decide to disregard, accept, or check equation validity using other methods.

\section{A priori Knowledge}

To some extent, it is possible to select a priori the type of models the algorithm will search for by selecting the functions to include in the function set. The choice of the function set is very important. A function set lacking a relevant function for the model may delay evolution or prevent it from finding a proper solution altogether. A way to take advantage of human expert knowledge is to seed the initial population of expressions with some we know are related with the problem. For example, when searching for the GDM we could have given the algorithm multiple forms of the ISAR to seed the search process. This is a directive approach and must be done in a parsimonious way, to avoid directing evolution too strongly, possibly trapping it in a local minimum. Such an approach should be complemented with random expressions in the initial population to create the necessary pool material for evolution to well explore the solution space. Therefore, a priori knowledge in SR has a stronger influence than in other inference methods, such as Bayesian, where a less adequate prior may be overcome by enough data.

\section{Fine-Tuning the Process}

The number of options in SR is immense. Population size is positively correlated with variability of models and how well the search space is explored, but might considerably slow the search. Mutation rates are also positively correlated with variability, but rates that are too high might prevent the algorithm converging on the best models. The fitness measure depends on the specific problem and on the type of noise in the data.
The number of generations to let the search run is entirely dependent on the problem complexity and time available. Often the algorithm reaches some equation that makes immediate sense to the researcher and the process can be immediately stopped for further analysis of results. Sometimes several competing equations seem to make sense but are not entirely convincing, in which case some indicators can be used as a stop rule, such as high values of stability and maturity of the evolution process (Schmidt, 2015).

The speed with which evolution occurs is extremely variable, depending on factors including the complexity of the relationships, having the appropriate variables and building blocks and the level of noise in the data. Fortunately, the process is easily adaptable to parallel computing, as many candidate functions can be evaluated simultaneously, allowing the use of multiple cores and even computer clusters to speed the search of equations.

\section{Caveats}

The SR approach is fully data-driven. This means it requires high-quality data if meaningful relationships are to be found. Also, it makes no a priori assumptions, so the final result might make no (obvious) sense, leading to spurious inferences, particularly if data are scarce or poor-quality, or if the right building blocks are not provided. Additionally, SR suffers from the same limitations of evolutionary algorithms in general. In many cases the algorithm may get stuck in local minima of the search space, requiring time (or even a restart with different parameters) to find the global minimum. Finally, SR suffers from the problem of bloat, which consists on an excessive growth of the expressions. There are mitigating approaches, like introducing a penalty for long expressions in the fitness function, or doing a posteriori symbolic analysis and simplification. However, bloat is still a problem under research.

Nevertheless, the fact that SR produces human legible expressions turns out to be useful even in the case of very large expressions. The expert eye can usually distinguish relevant fragments from a variety of unmeaningful segments of long expressions. And those relevant fragments often spur new thoughts and experiments.

\section{The Automation of Science?}

The methods here presented can be powerful additions to theoretical and experimental ecology, even if new conceptual hypotheses have to be created to accommodate the new equations. Such models could even be the only available means of investigating complex ecological systems when experiments are not feasible or datasets get too big/complex to model, using traditional statistical techniques (e.g., Tromas et al., 2017).

This family of techniques has led several authors to suggest the "automation of science" (King et al., 2009), where computers are able to advance hypotheses, test them, and reach conclusions in largely unassisted processes. This falls into the realm of exploiting knowledge (or symbolic) 
driven $\mathrm{AI}$ together with data driven $\mathrm{AI}$, or also automated machine learning an approach that recently began to gain momentum (e.g., Zhuang et al., 2017). SR potential is high in this automated science avenue since it bridges well from data to symbolic representations. What is clear already is its capability of producing formulas that help researchers to focus on initially imperceptible but interesting relationships within datasets and therefore SR may guide the process of hypothesis creation.

\section{DATA AVAILABILITY STATEMENT}

Eureqa scripts and Pareto front are available in Data Sheet 2. Data for Case study Modeling species richness are in Cardoso et al. (2013). Train and test datasets used for species distribution modeling are in Data Sheet 3. Dataset used in the case study Developing species richness estimators are available in the $\mathrm{R}$ package BAT (Cardoso et al., 2015). Dataset used in case study Developing the general dynamic model of oceanic island biogeography (GDM) are derived from Aranda et al. (2014) and Cardoso et al. (2010) see Data Sheet 4.

\section{AUTHOR CONTRIBUTIONS}

PC conceived the original idea and led the writing of the manuscript with LC. PC and VB performed the analyses with contributions from LC, JCC, and FR. PB and RG contributed with data. All authors contributed to the writing of the manuscript.

\section{REFERENCES}

Akaike, H. (1974). New look at statistical-model identification. Ieee T. Automat. Contr. 19, 716-723. doi: 10.1109/TAC.1974.1100705

Allouche, O., Tsoar, A., and Kadmon, R. (2006). Assessing the accuracy of species distribution models: prevalence, kappa and the true skill statistic (TSS). J. Appl. Ecol. 43, 1223-1232. doi: 10.1111/j.1365-2664.2006.01214.x

Almeida, J., dos Santos, J. A., Miranda, W. O., Alberton, B., Morellato, L. P. C., and Torres, R. D. (2015). Deriving vegetation indices for phenology analysis using genetic programming. Ecol. Inform. 26, 61-69. doi: 10.1016/j.ecoinf.2015.01.003

Anand, M., Gonzalez, A., Guichard, F., Kolasa, J., and Parrott, L. (2010). Ecological systems as complex systems: challenges for an emerging science. Diversity 2, 395-410. doi: 10.3390/d2030395

Aranda, S. C., Gabriel, R., Borges, P. A. V., Santos, A. M. C., de Azevedo, E. B., Patino, J., et al. (2014). Geographical, temporal and environmental determinants of bryophyte species richness in the Macaronesian islands. PLoS ONE 9:e101786. doi: 10.1371/journal.pone.0101786

Araújo, M. B., Anderson, R. P., Barbosa, A. M., Beale, C. M., Dormann, C. F., Early, R., et al. (2019). Standards for distribution models in biodiversity assessments. Sci. Adv. 5:eaat4858. doi: 10.1126/sciadv.aat4858

Barrett, J., Kostadinova, A., and Raga, J. A. (2005). Mining parasite data using genetic programming. Trends Parasitol. 21, 207-209. doi: $10.1016 /$ j.pt.2005.03.007

Barton, K. (2015). MuMIn: Multi-Model Inference. Available online at: https:// CRAN.R-project.org/package=MuMIn (accessed September, 2020).

Bertoni, R., Bertoni, M., Morabito, G., Rogora, M., and Callieri, C. (2016). A nondeterministic approach to forecasting the trophic evolution of lakes. J. Limnol. 75, 242-252. doi: 10.4081/jlimnol.2016.1374

\section{FUNDING}

PC and VB were supported by Kone Foundation. PB and FR were partly funded by the project FCT-PTDC/BIABIC/119255/2010 - Biodiversity on oceanic islands: toward a unified theory. LC was supported by FCT through LASIGE Research Unit, ref. UIDB, UIDP/00408/2020. SM acknowledges support from the European Commission through Horizon 2020 Marie Skłodowska-Curie Actions (MSCA) individual fellowships (Grant no. 882221).

\section{ACKNOWLEDGMENTS}

We thanked Robert Whittaker, Stano Pekár, Michael Lavine, and Otso Ovaskainen for comments on earlier versions of the manuscript; Carla Gomes and Ronan Le Bras for fruitful discussions around $\mathrm{AI}$ and ecology.

\section{SUPPLEMENTARY MATERIAL}

The Supplementary Material for this article can be found online at: https://www.frontiersin.org/articles/10.3389/fevo. 2020.530135/full\#supplementary-material

Data Sheet 1 | Examples of general principles in ecology and of some of the respective statistical models.

Data Sheet 2 | Data and settings used for all Symbolic Regression analyses in the paper (Eureqa file: http://www.nutonian.com/products/eureqa/).

Data Sheet 3 | Train and test databases used for species distribution modeling.

Data Sheet 4 | Species, area, and age for each Canarian Island.

Cardoso, P. (2009). Standardization and optimization of arthropod inventories-the case of Iberian spiders. Biodivers. Conserv. 18, 3949-3962. doi: 10.1007/s10531-009-9690-7

Cardoso, P., Aranda, S. C., Lobo, J. M., Dinis, F., Gaspar, C., and Borges, P. A. V. (2009). A spatial scale assessment of habitat effects on arthropod communities of an oceanic island. Acta Oecol. 35, 590-597. doi: 10.1016/j.actao.2009.05.005

Cardoso, P., Arnedo, M. A., Triantis, K. A., and Borges, P. A. V. (2010). Drivers of diversity in Macaronesian spiders and the role of species extinctions. J. Biogeogr. 37, 1034-1046. doi: 10.1111/j.1365-2699.2009.02264.x

Cardoso, P., Gaspar, C., Pereira, L. C., Silva, I., Henriques, S. S., da Silva, R. R., et al. (2008a). Assessing spider species richness and composition in Mediterranean cork oak forests. Acta Oecol. 33, 114-127. doi: 10.1016/j.actao.2007.10.003

Cardoso, P., Rigal, F., and Carvalho, J. C. (2015). BAT - Biodiversity Assessment Tools, an R package for the measurement and estimation of alpha and beta taxon, phylogenetic and functional diversity. Methods Ecol. Evol. 6, 232-236. doi: 10.1111/2041-210X.12310

Cardoso, P., Rigal, F., Fattorini, S., Terzopoulou, S., and Borges, P. A. V. (2013). Integrating Landscape disturbance and indicator species in conservation studies. PLoS ONE 8:e63294. doi: 10.1371/journal.pone.0063294

Cardoso, P., Scharff, N., Gaspar, C., Henriques, S. S., Carvalho, R., Castro, P. H., et al. (2008b). Rapid biodiversity assessment of spiders (Araneae) using semiquantitative sampling: a case study in a Mediterranean forest. Insect Conserv. Diver. 1, 71-84. doi: 10.1111/j.1752-4598.2007.00008.x

Chen, Y., Angulo, M. T., and Liu, Y. Y. (2019). Revealing complex ecological dynamics via symbolic regression. Bioessays 41:1900069. doi: 10.1002/bies.201900069

Cinalli, D., Martí, L., Sanchez-Pi, N., and Garcia, A. C. B. (2015). "Collective preferences in evolutionary multi-objective optimization: techniques and potential contributions of collective intelligence," in 30th Annual 
ACM Symposium on Applied Computing (New York, NY), 133-138. doi: $10.1145 / 2695664.2695926$

Clench, H. (1979). How to make regional lists of butterflies: some thoughts. J. Lepid. Soc. 33, 216-231.

Desjardins-Proulx, P., Poisot, T., and Gravel, D. (2019). Artificial intelligence and synthesis in ecology and evolution. Front. Ecol. Evol. 7:402. doi: $10.3389 /$ fevo.2019.00402

Dodds, W. K. (2009). Laws, Theories and Patterns in Ecology. Berkeley, CA: University of California Press. doi: 10.1525/california/9780520260405.001.0001

Dormann, C. F., Elith, J., Bacher, S., Buchmann, C., Carl, G., Carré, G., et al. (2013). Collinearity: a review of methods to deal with it and a simulation study evaluating their performance. Ecography 3, 27-46. doi: 10.1111/j.1600-0587.2012.07348.x

Dubčáková, R. (2011). Eureqa: software review. Genet. Program. Evol. M. 12, 173-178. doi: 10.1007/s10710-010-9124-Z

Elith, J., Graham, C. H., Anderson, R. P., Dudik, M., Ferrier, S., Guisan, A., et al. (2006). Novel methods improve prediction of species' distributions from occurrence data. Ecography 29, 129-151. doi: 10.1111/j.2006.0906-7590.04 596.x

Engelbart, D. (1962). Augmenting Human Intellect: A Conceptual Framework. Summary Report AFOSR-3233. Stanford Research Institute, Menlo Park, CA, United States. doi: 10.21236/AD0289565

Evans, M. R., Grimm, V., Johst, K., Knuuttila, T., de Langhe, R., Lessells, C. M., et al. (2013). Do simple models lead to generality in ecology? Trends Ecol. Evol. 28, 578-583. doi: 10.1016/j.tree.2013.05.022

Fattorini, S. (2009). On the general dynamic model of oceanic island biogeography. J. Biogeogr. 36, 1100-1110. doi: 10.1111/j.1365-2699.2009.02 083.x

Hastie, T. (2015). gam: Generalized Additive Models. Available online at: https:// CRAN.R-project.org/package=gam (accessed September, 2020).

Holland, J. H. (1975). Adaptation in Natural and Artificial Systems: An Introductory Analysis With Applications to Biology, Control, and Artificial Intelligence. Ann Arbor, MI: University of Michigan Press.

Holland, J. H. (1995). Hidden Order: How Adaptation Builds Complexity. Reading, MA: Addison-Wesley.

Holland, J. H. (1998). Emergence: From Chaos to Order. Reading, MA: AddisonWesley.

Hurvich, C. M., and Tsai, C. L. (1989). Regression and time series model selection in small samples. Biometrika 76, 297-307. doi: 10.1093/biomet/76.2.297

Jagupilla, S. C. K., Vaccari, D. A., Miskewitz, R., Su, T. L., and Hires, R. I. (2015). Symbolic regression of upstream, stormwater, and tributary E. coli concentrations using river flows. Water Environ. Res. 87, 26-34. doi: 10.1002/j.1554-7531.2015.tb00138.x

King, R. D., Rowland, J., Oliver, S. G., Young, M., Aubrey, W., Byrne, E., et al. (2009). The automation of science. Science 324, 85-89. doi: $10.1126 /$ science. 1165620

Koza, J. R. (1992). Genetic Programming: On the Programming of Computers by Means of Natural Selection. Cambridge, MA: MIT Press.

Larsen, P. E., Cseke, L. J., Miller, R. M., and Collart, F. R. (2014). Modeling forest ecosystem responses to elevated carbon dioxide and ozone using artificial neural networks. J. Theor. Biol. 359, 61-71. doi: 10.1016/j.jtbi.2014.05.047

Larsen, P. E., Field, D., and Gilbert, J. A. (2012). Predicting bacterial community assemblages using an artificial neural network approach. Nat. Methods 9, 621-625. doi: 10.1038/nmeth.1975

Lawton, J. H. (1996). Patterns in ecology. Oikos 75, 145-147. doi: 10.2307/3546237

Liu, C. R., Berry, P. M., Dawson, T. P., and Pearson, R. G. (2005). Selecting thresholds of occurrence in the prediction of species distributions. Ecography 28, 385-393. doi: 10.1111/j.0906-7590.2005.03957.x

Lu, Y. (2019). Artificial intelligence: a survey on evolution, models, applications and future trends. J. Manag. Anal. 6, 1-29. doi: 10.1080/23270012.2019.1570365

Manson, S. M. (2005). Agent-based modeling and genetic programming for modeling land change in the Southern Yucatan Peninsular Region of Mexico. Agr. Ecosyst. Environ. 111, 47-62. doi: 10.1016/j.agee.2005. 04.024

Manson, S. M., and Evans, T. (2007). Agent-based modeling of deforestation in southern Yucatan, Mexico, and reforestation in the Midwest United States. Proc. Natl. Acad. Sci. U.S.A. 104, 20678-20683. doi: 10.1073/pnas.070 5802104
Martin, B. T., Munch, S. B., and Hein, A. M. (2018). Reverse-engineering ecological theory from data. P Roy. Soc. B-Biol. Sci. 285:20180422. doi: $10.1098 / \mathrm{rspb} .2018 .0422$

Mitchell, M. (2009). Complexity: A Guided Tour. Oxford England, New York, NY: Oxford University Press.

Muttil, N., and Chau, K. (2006). Neural network and genetic programming for modelling coastal algal blooms. Int. J. Environ. Pollut. 28, 223-238. doi: 10.1504/IJEP.2006.011208

Muttil, N., and Lee, J. H. W. (2005). Genetic programming for analysis and real-time prediction of coastal algal blooms. Ecol. Model. 189, 363-376. doi: 10.1016/j.ecolmodel.2005.03.018

Nocedal, J., and Wright, S. J. (1999). Numerical Optimization. New York, NY: Springer. doi: 10.1007/b98874

Passy, S. I. (2012). A hierarchical theory of macroecology. Ecol. Lett. 15, 923-934. doi: 10.1111/j.1461-0248.2012.01809.x

Phillips, S. J., Anderson, R. P., and Schapire, R. E. (2006). Maximum entropy modeling of species geographic distributions. Ecol. Model. 190, 231-259. doi: 10.1016/j.ecolmodel.2005.03.026

R Core Team (2015). R: A Language and Environment for Statistical Computing. Vienna: R Foundation for Statistical Computing.

Ratkowsky, D. A. (1990). Handbook of Nonlinear Regression Models. New York, NY: M. Dekker.

Reshef, D. N., Reshef, Y. A., Finucane, H. K., Grossman, S. R., McVean, G., Turnbaugh, P. J., et al. (2011). Detecting novel associations in large data sets. Science 334, 1518-1524. doi: 10.1126/science.1205438

Russell, S. J., Norvig, P., and Davis, E. (2010). Artificial Intelligence: A Modern Approach, 3rd Edn. Upper Saddle River, NJ: Prentice Hall.

Schmidt, M., and Lipson, H. (2009). Distilling free-form natural laws from experimental data. Science 324, 81-85. doi: 10.1126/science.1165893

Schmidt, M. L. H. (2015). Eureqa. Available online at: https://www.nutonian.com/ (accessed September, 2020).

Smits, G. F., and Kotanchek, M. (2005). "Pareto-front exploitation in symbolic regression," in Genetic Programming Theory and Practice II, eds U.-M. O’Reilly, T. Yu, R. Riolo, and B. Worzel (Boston, MA: Springer), 283-299. doi: 10.1007/0-387-23254-0_17

Soberón, J., and Llorente, J. (1993). The use of species accumulation functions for the prediction of species richness. Conserv. Biol. 7, 480-488. doi: 10.1046/j.1523-1739.1993.07030480.x

Solé, R. V., and Goodwin, B. C. (2000). Signs of Life: How Complexity Pervades Biology. New York, NY: Basic Books.

Steinbauer, M. J., Klara, D., Field, R., Reineking, B., and Beierkuhnlein, C. (2013). Re-evaluating the general dynamic theory of oceanic island biogeography. Front. Biogeogr. 5, 185-194. doi: 10.21425/F5FBG19669

Sugihara, G., May, R., Ye, H., Hsieh, C. H., Deyle, E., Fogarty, M., et al. (2012). Detecting causality in complex ecosystems. Science 338, 496-500. doi: 10.1126/science.1227079

Triantis, K. A., Guilhaumon, F., and Whittaker, R. J. (2012). The island species-area relationship: biology and statistics. J. Biogeogr. 39, 215-231. doi: 10.1111/j.1365-2699.2011.02652.x

Tromas, N., Fortin, N., Bedrani, L., Terrat, Y., Cardoso, P., Bird, D., et al. (2017). Characterizing and predicting cyanobacterial blooms in an 8-year amplicon sequencing time course. ISME J. 11, 1746-1763. doi: 10.1038/ismej.2017.58

Tung, C. P., Lee, T. Y., Yang, Y. C. E., and Chen, Y. J. (2009). Application of genetic programming to project climate change impacts on the population of Formosan Landlocked Salmon. Environ. Modell. Softw. 24, 1062-1072. doi: 10.1016/j.envsoft.2009.02.012

Whittaker, R. J., Triantis, K. A., and Ladle, R. J. (2008). A general dynamic theory of oceanic island biogeography. J. Biogeogr. 35, 977-994. doi: 10.1111/j.1365-2699.2008.01892.x

Yanco, H. A., Drury, J. L., and Scholtz, J. (2004). Beyond usability evaluation: analysis of human-robot interaction at a major robotics competition. HumComput. Interact. 19, 117-149. Available online at: https://www.nist.gov/ publications/beyond-usability-evaluation-analysis-human-robot-interactionmajor-robotics-competition

Yao, M. J., Rui, J. P., Li, J. B., Dai, Y. M., Bai, Y. F., Hedenec, P., et al. (2014). Rate-specific responses of prokaryotic diversity and structure to nitrogen deposition in the Leymus chinensis steppe. Soil Biol. Biochem. 79, 81-90. doi: 10.1016/j.soilbio.2014.09.009 
Zhuang, Y., Wu, F., Chen, C., and Pan, Y. (2017). Challenges and opportunities: from big data to knowledge in AI 2.0. Front. Inform. Tech. El. 18, 3-14. doi: 10.1631/FITEE.1601883

Conflict of Interest: The authors declare that the research was conducted in the absence of any commercial or financial relationships that could be construed as a potential conflict of interest.
Copyright (C) 2020 Cardoso, Branco, Borges, Carvalho, Rigal, Gabriel, Mammola, Cascalho and Correia. This is an open-access article distributed under the terms of the Creative Commons Attribution License (CC BY). The use, distribution or reproduction in other forums is permitted, provided the original author(s) and the copyright owner(s) are credited and that the original publication in this journal is cited, in accordance with accepted academic practice. No use, distribution or reproduction is permitted which does not comply with these terms. 